Acta Physica Academiae Scientiarum Hungaricae, Tomus 24 (4), pp. 337-345 (1968)

\title{
ИСПОЛЬЗОВАНИЕ ПОВЕРХНОСТНЫХ УПРУГИХ КОЛЕБАНИЙ ДЛЯ ИЗМЕРЕНИЯ НЕКОТОРЫХ ХАРАКТЕРИСТИК ПОЛУПРОВОДНИКОВЫХ ПЛЕНОК
}

\author{
С. В. БОГДАНОВ и И. Б. ЯКОВКИН \\ ИНСТИТУТ ФИЗИКИ ПОЛУПРОВОДНИКОВ АН СССР, НОВОСИБИРСК, СССР
}

(Представлено Г. Сигети. - Поступило 30. V. 1967)

\begin{abstract}
Авторы используют поверхностные упругие колебания для измерения характеристик полупроводниковых пленок. Исследования проводились на ориентированных пленках CdS. Авторами установлено воздействие адсорбированных газов и других факторов.
\end{abstract}

1.Упругие поверхностные колебания привлекают все большее внимание исследователей как инструмент для исследования поверхности твердого тела. Свойства многих полупроводниковых приборов в большой степени определяются поверхностным состоянием материала: концентрацией свободных носителей, их подвижностью, концентрацией дефектов и дислокаций, адсорбированными поверхностью примесями и т. д. Параметры упругой волны, возбужденной на поверхности твердого тела, в свою очередь зависят от состояния поверхности. Таким образом, исследования условий распространения поверхностных волн существенно расширяют информацию о состоянии поверхности твердого тела.

В данной работе основное внимание уделялось изучению затухания упругих поверхностных волн.

При исследовании распространения упругих релеевских волн в системе подложка-пленка, для оценки затухания, вносимого пленкой, необходимо знать распределение плотности кинетической энергии волны между пленкой и подложкой. Выполнить подобный расчет вполне строго оказывается чрезвычайно сложным, т. к. в общем случае упругие константы пленки и материала подложки различны и на границе раздела подложка-пленка будут иметь место различные граничные эффекты. Однако, если толщина пленки много меньше длины волны, то из обших физических соображений ясно, что наличие пленки не может существенно сказываться ни на структуре волны, ни на распределении плотности ее энергии с глубиной. Т. е. в этом случае не важно, каковы собственные упругие параметры пленки, и их можно положить такими же как и в материале подложки. Тақим образом, задача фактически сводится К нахождению плотности кинетической энергии поверхностной волны в однородной среде.

Полученное распределение плотности кинетической энергии по глубине может быть использовано как для определения затухания в массивном образ- 
це из материала пленки, так и для определения толщины пленки по измеренному затуханию в системе подложка-пленка.

2. Нами выполнены численные расчеты для распределения плотности кинетической энергии в приповерхностном слое для материалов с различными коэффициентами Пуассона - $v$.

Расчетные данные представлены на рис. 1 ввиде относительных кривых распределения плотности кинетической энергии релеевской волны. Здесь кривые: 1 - для плавленного кварца; 2 - сульфида кадмия, 3 - антимонида

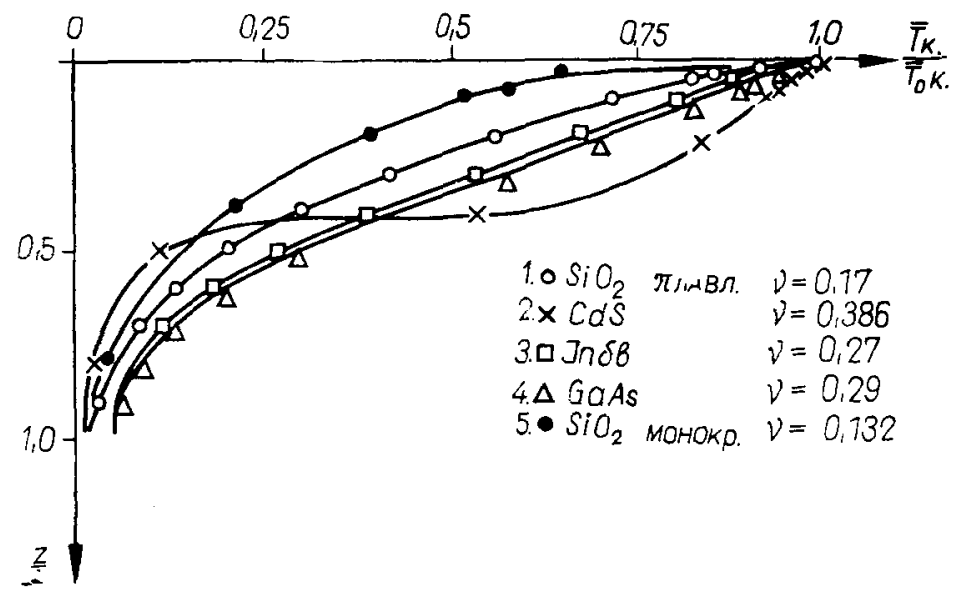

Pис. 1. Распределение плотности кинетической энергии поверхностной волны в зависимости от расстояния до поверхности, изотропный случай

$\begin{array}{ll}\text { 1. } \mathrm{SiO}_{2}, & y=0,17 \\ \text { 2. } \mathrm{CdS}, & y=0,386 \\ \text { 3. InSb, } & y=0,27 \\ \text { 4. } \mathrm{GaAs} & y=0,29 \\ \text { 5. } \mathrm{SiO}_{2}, & y=0,132\end{array}$

индия, 4 - арсенида галлия, 5 - кварца с $v=0,132, \lambda=$ длина релеевской волны.

Из данных, представленных на рис. 1 видно, что максимальная плотность кинетической энергии сосредоточена в узком приповерхностном слое. Это позволяет надеяться, что наличие тонкой пленки на поверхности или состояние самой поверхности, отличное от состояния объема, должно определенным образом сказываться на затухание поверхностных волн.

3. Рассмотрим модель системы подложка-пленка, по которой распространяется поверхностная волна. Толшина пленки предполагается много меньше длины упругой волны с тем, чтобы наличие пленки не приводилок изменению типа колебаний. Зная распределение плотности кинетической энергии, можно отыскать затухание в системе подложка-пленка $\mathrm{CdS}$, считая, что затухание упругих колебаний пропорционально кинетической 
части полной энергии, и учитывая перераспределение энергии между подложкой и пленкой по мере распространения волны.

Коэффициент затухания поверхностной волны в материале пленки $\alpha_{m}$ можно представить в виде:

$$
x_{m}=\frac{\Delta x}{l} \cdot \frac{\int_{0}^{\infty} \bar{T}_{k} d z}{\int_{0}^{h} \bar{T}_{k} d z} \simeq 0,18 \frac{\Delta \alpha}{l} \cdot \frac{\lambda}{h},
$$

где: $\bar{T}_{K}-$ плотность кинетической энергии,

$\Delta \alpha-$ затухание, вносимое пленкой, толщиной $h$ и длиной $l$,

$\lambda$ - длина волны.

Выражение (1) получено для подложки из плавленного кварца в предположении, что коэффициент затухания в подложке много меньше $\alpha_{m}$.

Полученное выражение позволяет по известным $\Delta x, \lambda, l$ определить либо толщину пленки $\boldsymbol{h}$, если известно $\alpha_{m}$, (например, в процессе ее напыления в вакууме), либо $\alpha_{m}$, если известно $h$ [1].

4. Основываясь на предположении, что состояние поверхности должно сқазываться на затухании релеевских волн, представляло интерес осуществить следующие эксперименты:

1) возбуждать поверхностную упругую волну на кварцевой подложке как в атмосфере воздуха, так и в вакууме;

2) исследовать затухание поверхностной волны в системе подложкапленка:

а) зависимость затухания от толщины пленки;

б) зависимость затухания от проводимости пленки (фоточувствительное затухание);

в) влияние адсорбированных газов на затухание релеевских волн в системе подложка-пленка.

Эксперименты проводились с ориентированными пленками CiS толщиной от 0,1 до 18 микрон и длиной до $6 \mathrm{~cm}$. Удельная проводимость пленок при дневном освещении составляла величину порядка $10^{-4}-10^{-6} \mathrm{om}^{-1} \mathrm{~cm}^{-1}$ и слабо зависела от освещения.

В процессе всех экспериментов снималась проходная характеристика системы, т. е. затухание упругих волн в зависимости от амплитуды радиочастотного импульса, возбуждающего релеевскую волну.

5. а) Влияние атмосферы на затухание упругой поверхностной волны в кварцевой подложке.

Система возбуждения и регистрация релеевских волн помещалась в вакуумный объем с давлением остаточных газов $1 \cdot 10^{-7}$ торр. Полное затухание поверсностных волн на свободной предварительно прогретой подложке составляло в этом случае величину 60,5 дб. Затем в вакуумный объем напус- 
кался воздух. При этом затухание в системе практически не изменялось. Таким образом, изменение затухания релеевской волны в кварцевой подложке, вызванное наличием адсорбированных газов, лежит ниже чувствительности использованной нами аппаратуры.

б) Затухание упругой поверхностной волны в системе подложкапленка.

При напылении пленки CiS на кварцевую подложку затухание в системе заметно возрастало. Результаты экспериментов для 5-и пленок различной толщины, напыленных на плавленный кварц и имевших при дневном освещении проводимость $\sigma \sim 10^{-5} \mathrm{om}^{-1} \mathrm{~cm}^{-1}$, представлены в табл. 1. Здесь же даны значения коэффициента затухания $x_{m}$ релеевских волн в массивном образце, рассчитанные по выражению (1).

Таблица 1

\begin{tabular}{|c|c|c|c|c|c|c|}
\hline $\begin{array}{c}\text { № № } \\
\text { ПI/П }\end{array}$ & $\mid \begin{array}{c}\text { Частота звука } \\
f \\
(\text { Maц })\end{array}$ & $\begin{array}{c}\text { Толщина } \\
\text { пленки } \\
h \\
(\mu)\end{array}$ & $\mid \begin{array}{c}\text { Длина пленки } \\
l \\
(с M)\end{array}$ & $\begin{array}{c}\text { Проводимость } \\
\text { Пленки } \\
\sigma \\
\left(\text { OM-1 }^{-1} \cdot \mathrm{cM}^{-1}\right)\end{array}$ & $\begin{array}{c}\text { Вносимое } \\
\text { затухание } \\
\Delta \alpha \\
(\partial \sigma)\end{array}$ & $\begin{array}{c}\text { Коэффицнент } \\
\text { затухания в } \\
\text { массивном } \\
\text { образце } \\
\alpha_{m} \\
(\partial \sigma / c M)\end{array}$ \\
\hline 1 & 21,0 & 0,7 & 5 & $10^{-5}$ & 1,5 & 14 \\
\hline 2 & 21,0 & 1,5 & 5 & $10^{-5}$ & 4,0 & 11 \\
\hline 3 & 21,0 & 3 & 5 & $10^{-5}$ & 5,5 & 12 \\
\hline 4 & 21,0 & 5,3 & 3 & $10^{-5}$ & 7,8 & 13 \\
\hline 5 & 21,0 & 4,0 & 4 & $10^{-5}$ & 8,3 & 14 \\
\hline
\end{tabular}

Представляет интерес сопоставить эти значения $\alpha_{m}$ с результатами прямых измерений қоэффициента затухания релеевских волн в массивных образцах $\mathrm{CdS}[3]$, а также с расчетным, найденных согласно [2, 4]. Эти данные приведены в таблице 2.

Таблица 2

\begin{tabular}{|c|c|c|c|c|}
\hline Материал & $\begin{array}{l}\text { Скорость пов. } \\
\text { волн см/сек. }\end{array}$ & $\begin{array}{l}\text { Коэф. зат. пов. } \\
\text { волн дб/см }\end{array}$ & Частота Mru & Примечание \\
\hline $\begin{array}{l}\text { CdS } \\
\text { в напр } \perp \text { оси } \\
Z\end{array}$ & & 13 & 21 & $\begin{array}{l}\text { Рассчитан по измеренному затуханию } \\
\text { в тонких пленках } 0,7-3 \mu \\
\left(\sigma \sim 10^{-5} \text { ом }^{-1} \mathrm{~cm}^{-1}\right. \\
\text { на плавленном } \mathrm{SiO}_{z}\end{array}$ \\
\hline $\begin{array}{l}\text { CdS } \\
\text { в напр } \perp \text { оси } \\
Z\end{array}$ & $1,7 \cdot 10^{5}$ & $10 \ldots 20$ & 25,0 & $\begin{array}{l}\text { Расчетное значение для массив- } \\
\text { ного образца } \\
\left(\sigma \sim 10^{-5} \mathrm{om}^{-1} \mathrm{~cm}^{-1}\right)[2,4]\end{array}$ \\
\hline ", & $"$, & 10,0 & 30 & $\begin{array}{l}\text { Измерено на массивном образце при } \\
\sigma \sim 10^{-5} \mathrm{om}^{-1} \mathrm{~cm}^{-1}[3]\end{array}$ \\
\hline
\end{tabular}


Как видно из таблицы, затухание упругой поверхности волны в массивном образце $\mathrm{CdS}$, рассчитанное по измеренному затуханию в тонких пленках, вполне удовлетворительно согласуется как с расчетным значением по [2, 4], так и с измеренным экспериментально [3]. Приняв затухание поверхностных волн в массивном образце $\alpha_{m}=13$ дб/см, по выражению (1) можно найти толщину пленки $h$.На рис. 2 представлена зависимость $\Delta \alpha / l=f(h)$

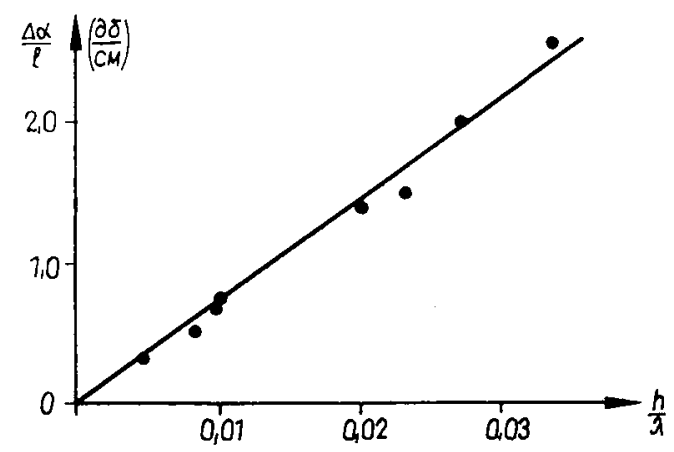

Puc. 2. Зависимость $\Lambda \alpha / l=f(h)$

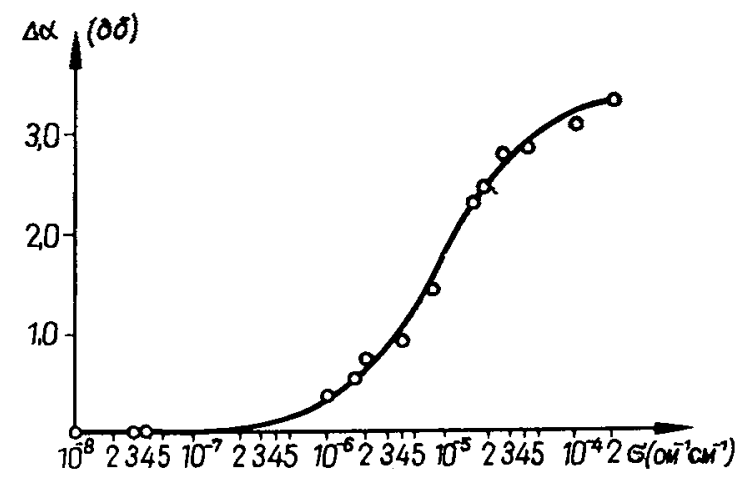

Puc. 3. Зависимость затухания $\Delta \alpha$ упругой поверхностной волны от проводимости $\sigma$

согласно (1) (сплошная линия). На этом же рис. точками отмечены экспериментально полученные значения для диапазона частот $5 \ldots 21$ Мгц.

в) Фоточувствительное затухание поверхностных волн в системе подложка-пленка; влияние адсорбированных газов.

Как уже отмечалось, большинство пленок, полученных на подложках из плавленного кварца, заметной фотопроводимостью не обладало. Однако, на подложках из кристаллического кварца в ряде случаев были получены пленки, удельная проводимость которых изменялась от $10^{-8} \mathrm{om}^{-1} \mathrm{~cm}^{-1}$ в темноте до $10^{-4} \mathrm{om}^{-1} \mathrm{~cm}^{-1}$ на свету. Эти пленки позволяли проследить изменение затухания упругой поверхностной волны в системе подложка-пленка при изменении удельной проводимости пленки. 
Результаты этих экспериментов представлены на рис. 3, где вызванное освещением приращение общего затухания в системе (на воздухе) изображено как функция проводимости пленки, т. е.

$$
\delta \alpha=\Delta \alpha_{o c \beta}-\Delta \alpha_{m e M H}=f\left(\sigma_{n \Omega}\right)
$$

Как видно из рисунка в диапазоне проводимостей от $10^{-8}$ до $10^{-6}$ ом $^{-1}$ $\mathrm{cm}^{-1}$ затухание поверхностных весьма незначительно. Максимальное изменение затухания соответствует области проводимости $10^{-5}-10^{-4} \mathrm{om}^{-1} \mathrm{~cm}^{-1}$. Такой ход фоточувствительного затухания релеевских волн в CCS corласуется с экспериментальными данными проводимыми в работе [3].

Сравнение фоточувствительного затухания пленок, находящихся в вакууме, а затем - на воздухе (после напускания воздуха в вакуумную систему), позволяло оценить влияние адсорбированных газов на проводимость полученных пленок.

Прямые измерения затухания поверхностной волны в системе подложкапленка в вакуумном объеме показали, что вносимое пленкой затухание при данной величине подсветки зависит от наличия адсорбированных пленкой газов: если в темноте вносимое пленкой затухание не зависит от наличия адсорбированных газов (в наших экспериментах величина $\Delta \alpha$ составляла примерно 9,0 дб), то на свету вносимое пленкой затухание возрастало примерно до 16,5 д6, если пленка находилась в вакууме, и всего до 12,5 дб, если пленка находилась на воздухе. Таким образом, в первом случае (вакуум) освещение вызывапо изменение затухания в системе на 7,5 дб, а во втором слууае (воздух) всего на 3,5 дб.

Проходные харақтеристики, соответствующие вышеперечисленным экспериментам представлены на рис. 4. Первая кривая - для свободной подложки, вторая - для подложки с пленкой C.S в темноте (в вакууме и после напуска воздуха), третья - для освещенной подложки с CaS в вакууме, четвертая - для освещенной подложки с CoS после напуска воздуха.

Все же из приведенных выше данных можно сделать вывод, что при фиксированной интенсивности света проводимость пленки в вакууме была больше, чем на воздухе. Это легко объяснить влиянием адсорбированных газов, если допустить, что молекулы адсорбированного газа являются «ловушками", захватывающими электроны и тем самым снижающими обшую проводимость пленки.

Влияние подсветки и адсорбированных газов на затухание поверхностных упругих колебаний в системе подложка пленка свидетельствуют 06 электронном характере затухания. Оно обусловлено взаимодействием электронов проводимости с электрическим полем в пленке. Это поле вызвано жак пьезоэффектом самой пленки, так и пьезоэффектом подложки. Предоставляло интерес разделить влияние пьезоэлектрического поля подложки (моно- 
$\mathrm{SiO}_{2}$ ) и пленки сульфида кадмия. $\mathrm{C}$ этой целью исследовалось фоточувствительное затухание поверхностных волн в системе с подложкой из плавленного кварца.

Проходные характеристики для этого случая представлены на рис. 5. Здесь для пленки толщиной $2,3 \mu$ сплошная кривая 1 - темновое затухание в системе, 2 - затухание на свету сразу после напуска воздуха, 3 - то же по

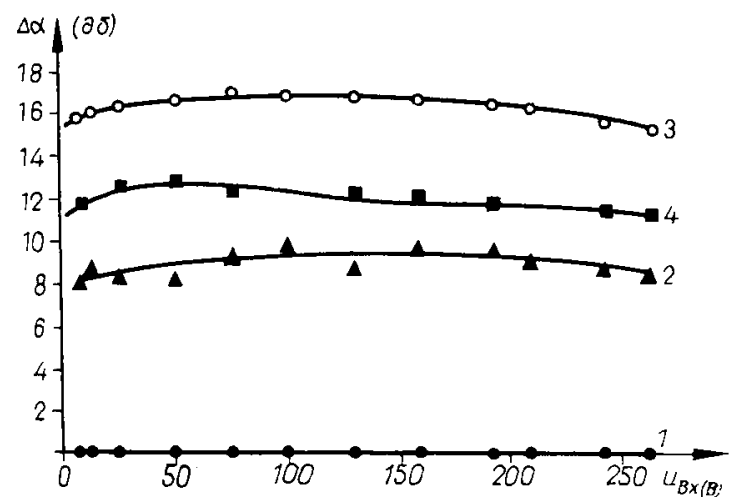

Puc. 4. Проходная характеристика системы: подложка-пленка CdS 1. Свободная подложка. 2. Подложка $c$ пленкой CdS в вакууме и воздухе в темноте. 3. Подложка с пленкой $\mathrm{CdS}$ в вакууме, без освешения. 4. Подложка в воздухе, освещенная

Puc.5. Проходные характеристики для системы подложка (плавленный кварц)

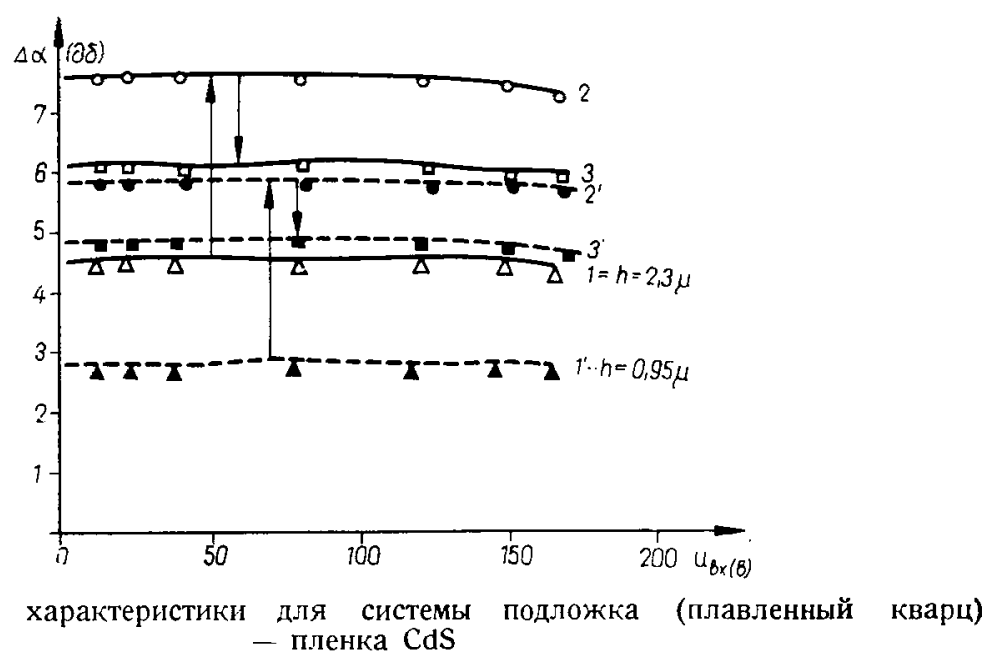

прошествии 3-х суток; для пленки толщиной $0,95 \mu$, пунктирная кривая $-\mathrm{l}^{\prime}-$ темновое затухание, $2^{\prime}$ - затухание на свету, $3^{\prime}$ - затухание на свету, по прошествии 3-х суток на воздухе. Из сравнения рисунков 4 и 5 видно, что фоточувствительное затухание в системе с подложкой из кристаллического 
кварца примерно в 5 раз больше, чем в системе с подложкой из плавленного кварца, что несомненно свидетельствует о влиянии пьезоэлектрических свойств подложки, поскольку ориентация пленки $\mathrm{CdS}$ на плавленном кварце была не хуже, чем на монокристаллическом.

Проведенные исследования электронного затухания упругих поверхностных волн в системе кристаллический кварц-фотопроводящая пленка показывают, что по величине затухания можно судить о фотопроводимости

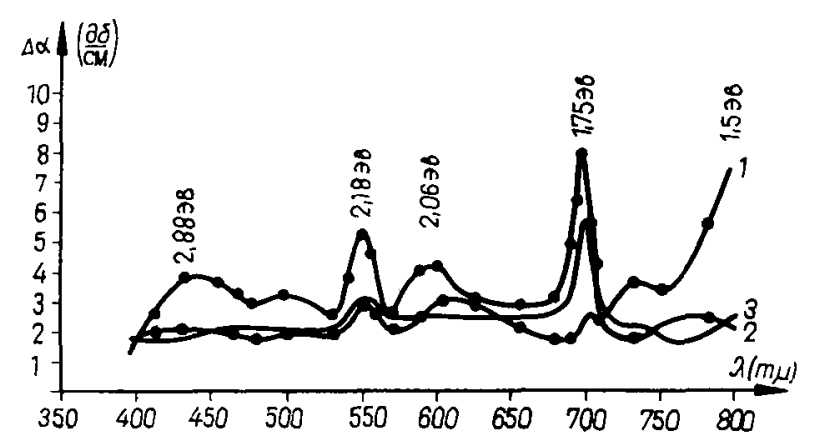

Puc. 6. Спектральная характеристика фоточувствительного затухания

пленки. Это открывает возможность бесконтактного изменения спектра фотопроводимости пленок. Результаты измерения спектра фотопроводимости сульфида кадмия в диапазоне длин волн света от 400 до $800 \mathrm{~m} \mu$ представлена на рис. 6 , где отчетливо видны пики поглощения соответсвующие примесным уровням. Кривая - 1 для $\mathrm{C}$ CS, напыленного из материала с большим содержанием примесей, кривые 2 и 3 из CذS любезно представленным нам профессором Э. Ж. Грийо (Сорбона), соответственно: с минимальным содержанием примесей, с примесью серебра.

6. Авторы пользуются случаем выразить свою признательность професcopy Э. Ж. Грийо за предоставления материала для исследований, а так же сотрудникам лаборатории М. К. Балакиреву за полезные дискуссии, Г. А. Лобановой и А. М. Жарову за помощь в проведении экспериментов.

\section{ЛИТЕРАТУРА}

1. С. В. Богданов и И. Б. Яковкин, Затухание упругих поверхностных волн в системе подложка-пленка. Акуст. журнал (напр. в печать).

2. F. Press and J. Healy, Journ. Appl. Phys., 28, 1323, 1957.

3. И. А. Викторов, Акустический журнал, Х1I, 251, 1966.

4. A. А. Морозов, ФТT, 7, 324, 1965. 


\section{APPLICATION OF SURFACE ELASTIC VIBRATIONS FOR THE MEASUREMENT OF SOME CHARACTERISTICS OF SEMICONDUCTOR FILMS}

S. V. BOGDANOV and I. B. YAKOVKIN

$$
\text { A bstract }
$$

The authors use surface elastic vibrations to determine some physical characteristics of semiconductor films. The investigations have been carried out on CdS oriented films. The effect of adsorbed gases and other factors is considered. 